\section{International Scientific Journal Theoretical \& Applied Science}

p-ISSN: 2308-4944 (print) e-ISSN: 2409-0085 (online)

Year: $2016 \quad$ Issue: 4 Volume: 36

Published: $30.04 .2016 \quad \underline{\text { http://T-Science.org }}$

SECTION 20. Medicine.
Malika Berikkyzy Maimakhova Master`s Student in Medical Sciences International Kazakh-Turkish University by name Yassavi, Kazakhstan m.maimakhova@gmail.com

Kumisai Sabetovna Babayeva Candidate of medical science, a.e. association professor Head of the Department of Emergency Medical Care International Kazakh-Turkish University by name Yassavi, Kazakhstan atu1972@mail.ru

Assel Dauletbaevna Sadykova $\mathrm{PhD}$ Student in Medicine Sciences International Kazakh-Turkish University by name Yassavi, Kazakhstan asikosha@inbox.ru

Murat Asylbekovich Shegebaev Senior lecturer International Kazakh-Turkish University by name Yassavi, Kazakhstan karadias77@mail.ru

Zhanar Sailaubekovna Shalkharova DM, Vice-president GI "Educational and Healthcare Holding" zhanar.shalkharova@gmail.com

Zhanat Nagmetovna Shalkharova DM, Professor International Kazakh-Turkish University by name Yassavi, Kazakhstan janatsh@mail.ru

\title{
FOOD HABIT STUDY IN PATIENTS WITH CHRONIC GASTRITIS, LIVING IN TURKESTAN REGION (SOUTH KAZAKHSTAN)
}

Abstract: The article presents the features of the feeding habits of people with chronic gastritis and the daily consumption of nutrients, microelements and vitamins.

Key words: food habit, chronic gastritis, nutrients, microelements, vitamins.

Language: English

Citation: Maimakhova MB, Babaeva KS, Sadykova AD, Shegebaev MA, Shalkharova ZN, Shalkharova ZS, (2016) FOOD HABIT STUDY IN PATIENTS WITH CHRONIC GASTRITIS, LIVING IN TURKESTAN REGION (SOUTH KAZAKHSTAN). ISJ Theoretical \& Applied Science, 04 (36): 153-157.

Soi: http://s-o-i.org/1.1/TAS-04-36-26 Doi: crossef http://dx.doi.org/10.15863/TAS.2016.04.36.26

BACKGROUND: Gastrointestinal diseases have a tendency for increasing day to a day. Consequently, gastrointestinal diseases define as an important problem in clinics nowadays, in which clinicians and public health care system are interested in. The main causes of these diseases are such factors as: unbalanced diet, stress, unfavorable environmental conditions and infectious agents [1].

Incidences of gastrointestinal diseases are on the third place after cardiovascular diseases. Gastritis is the most common pathology from all gastrointestinal diseases and $50 \%$ residents of Kazakhstan suffer from it [2]. In the majority countries of the world nearly $80-90 \%$ of people suffer from gastritis, and moreover, atrophic gastritis, also named as "premalignant case" is defined 5\% in people <30 years, $30 \%$ in $31-50$ years old and $50-70 \%$ in older people, upon 50 years $[3,4]$.

Researchers from Germany looking at food habits of elderly people revealed, that in elder age 


\begin{tabular}{|c|c|c|c|c|c|c|}
\hline Impact Factor: & $\begin{array}{l}\text { ISRA (India) } \\
\text { ISI (Dubai, UAB } \\
\text { GIF (Australia) } \\
\text { JIF }\end{array}$ & $\begin{array}{r}=1.344 \\
=0.829 \\
=0.564 \\
=1.500\end{array}$ & $\begin{array}{l}\text { SIS (USA) } \\
\text { PИHЦ (Russia) } \\
\text { ESJI (KZ) } \\
\text { SJIF (Morocco) }\end{array}$ & $\begin{array}{l}=0.912 \\
=0.234 \\
=1.042 \\
=2.031\end{array}$ & $\begin{array}{l}\text { ICV (Poland) } \\
\text { PIF (India) } \\
\text { IBI (India) }\end{array}$ & $\begin{array}{l}=6.630 \\
=1.940 \\
=4.260\end{array}$ \\
\hline
\end{tabular}

there is often a deficit of vitamins B12 and D3 in a combination of chronic diseases and psych emotional problems. The most frequent cause of deficit of vitamin B12 was the result of malabsorption against a background of atrophic gastritis [6].

In researches, investigated in Kazan revealed a lack of carbohydrates and energy in a nutrition, which made developing chronic gastroduodenitis in youth man, when in youth women it was found a deficiency of proteins, carbohydrates and daily calories [7].

Explorers from Brazil identified link between chronic gastritis, related H. Pylori had more lower level of vitamin B12 [8]. The study made by Naja F., Kreiger N., Mc. Keown, Eyssen G., Allard J., and can suppose that H. Pylori decrease biological availableness of vitamins $\mathrm{E}$ and $\mathrm{C}$ [9]. Other researchers: Ryz N.R. and Lochner A., investigated the level of vitamin D and revealed, that the lack of vitamin $\mathrm{D}$ make critical an intestinal inflammatory reactions on infectious, also reducing immune system, makes an organism more sensitive to chronic gastritis and other gastrointestinal diseases [10]. The result of next issue tells us about reducing abilities of vitamin $\mathrm{D}$ to an invasive intestinal flora, which influence on a mucous of gastrointestinal tract [10]. There is an another work, the result of which tell us that vitamin $\mathrm{D}$ can reduce unhealthy influence invasive intestinal flora, which impact on a mucous of gastrointestinal tract, supporting it. Thus, authors think, that deficiency of vitamin D predispose to developing for gastrointestinal diseases [11]. In Russia there was explored the link between microelements such as $\mathrm{Zn}$, Se, Mn with chronic gastritis. As the result they revealed, that lack of these microelements predispose to developing atrophic changes in mucous of a stomach [12].

AIM: To study the prevalence of chronic gastritis and to estimate the link between the food diet in Turkestan region residents.

METHODS: The research was in a Turkestan region among 1141 people, average age of whom was $51,9 \pm 13,7$. There was questioning for studying food diet. Data of questioning was calculated with the help of online-calculator [http://health-diet.ru/]. With the help of this calculator there were identified calories, pies of proteins, carbohydrates, lipids, microelements, depending on gender, age, weight and the level of physical activity. Statistical program SPSS Statistics 17.0 was used. For comparing average points it was used Student's criteria.

RESULTS: In the result we revealed 122 respondents, suffered from chronic gastritis, 46 of them were- man, and women were 76 , respectively. There was the most frequent prevalence of chronic gastritis in 60-74 age categories - among women (fig.1).

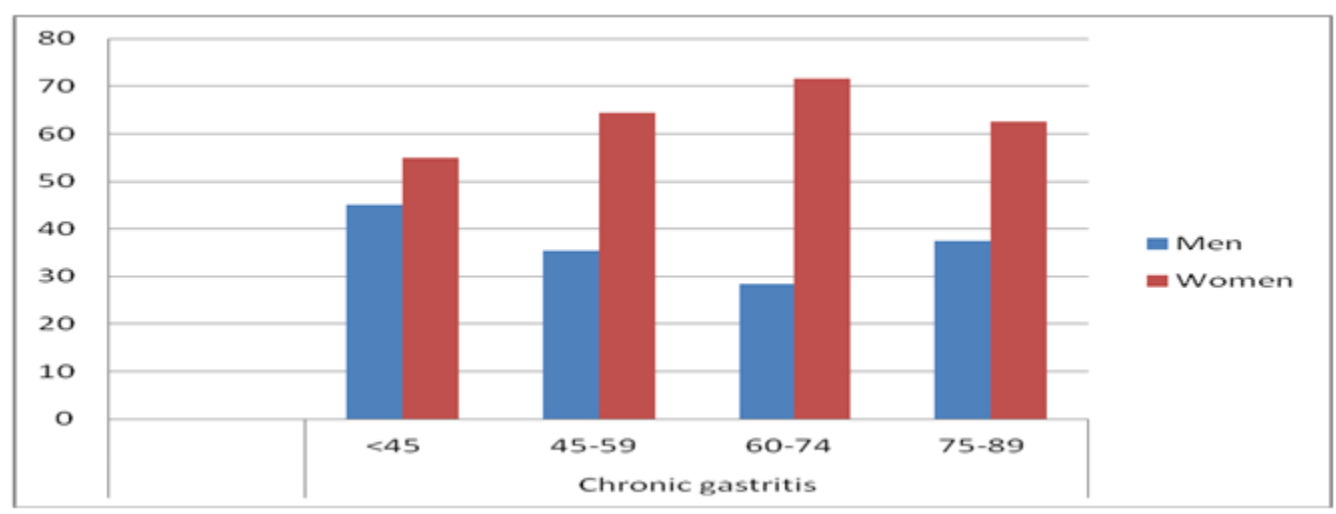

Figure1 - Prevalence of chronic gastritis, depending on gender and age.

Table 1

Comparing average trades of nutrients in patients with chronic gastritis and control group.

\begin{tabular}{|c|c|c|c|}
\hline Nutrients & $\begin{array}{c}\text { Chronic gastritis } \\
\mathrm{n}=\mathbf{1 2 2} \\
\mathrm{M}(\mathrm{SD}) \\
\end{array}$ & $\begin{array}{c}\text { Healthy people } \\
\mathbf{n}=\mathbf{1 0 0} \\
\mathrm{M}(\mathrm{SD}) \\
\end{array}$ & $\mathbf{p}$ \\
\hline Calories & $143,5( \pm 72,9)$ & $120,8( \pm 38,9)$ & 0,006 \\
\hline Proteins & $127,2( \pm 95,5)$ & $108,9( \pm 39,7)$ & 0,075 \\
\hline Fat & $140,4( \pm 85,03)$ & $116,04( \pm 36,5)$ & $\mathbf{0 , 0 0 8}$ \\
\hline Carbohydrates & $151,4( \pm 66,8)$ & $125,1( \pm 32,4)$ & 0,0001 \\
\hline Water & $46,07( \pm 14,4)$ & $58,2( \pm 17,8)$ & 0,0001 \\
\hline
\end{tabular}

ISPC Global Science, 


\begin{tabular}{l|lr|ll|ll} 
& ISRA (India) & $=\mathbf{1 . 3 4 4}$ & SIS (USA) & $=\mathbf{0 . 9 1 2}$ & ICV (Poland) & $=\mathbf{6 . 6 3 0}$ \\
Impact Factor: & ISI (Dubai, UAE) $=\mathbf{0 . 8 2 9}$ & PUH (Russia) & $=\mathbf{0 . 2 3 4}$ & PIF (India) & $=\mathbf{1 . 9 4 0}$ \\
& GIF (Australia) & $=\mathbf{0 . 5 6 4}$ & ESJI (KZ) & $=\mathbf{1 . 0 4 2}$ & IBI (India) & $\mathbf{4 . 2 6 0}$ \\
& JIF & $=\mathbf{1 . 5 0 0}$ & SJIF (Morocco) & $\mathbf{2 . 0 3 1}$ & & \\
\hline
\end{tabular}
Dietary Fibers $127,6( \pm 66,7)$ $111,2( \pm 40,5)$ 0,032

The results showed us, that in people with chronic gastritis there were statistically significant trades of calories, lipids and carbohydrates, comparing to control group.

Table 2

Comparing average trades of vitamins level in patients with chronic gastritis and control group.

\begin{tabular}{|c|c|c|c|}
\hline Vitamins & $\begin{array}{c}\text { Chronic gastritis } \\
\mathbf{n}=\mathbf{1 2 2} \\
\mathbf{M}(\mathrm{SD})\end{array}$ & $\begin{array}{c}\text { Healthy people } \\
\mathbf{n}=\mathbf{1 0 0} \\
\text { M(SD) }\end{array}$ & p \\
\hline b-car & $3,8( \pm 9,3)$ & $8,8( \pm 13,5)$ & 0,001 \\
\hline $\mathbf{A}$ & $223,9( \pm 171,02)$ & $129,08( \pm 53,6)$ & 0,0001 \\
\hline B1 & $72,1( \pm 29,6)$ & $78,05( \pm 25,7)$ & 0,120 \\
\hline B2 & $108,1( \pm 107,9)$ & $87,2( \pm 60,2)$ & 0,085 \\
\hline B5 & $62,1( \pm 28,5)$ & $75,3( \pm 23,5)$ & 0,0001 \\
\hline B6 & $80,1( \pm 43,6)$ & $89,09( \pm 27,7)$ & 0,075 \\
\hline B9 & $38,2( \pm 13,9)$ & $57,4( \pm 28,6)$ & 0,0001 \\
\hline B12 & $93,7( \pm 90,2)$ & $97,9( \pm 61,5)$ & 0,696 \\
\hline $\mathrm{C}$ & $50,3( \pm 33,03)$ & $68,4( \pm 31,4)$ & 0,0001 \\
\hline $\mathbf{E}$ & $96,2( \pm 53,8)$ & $81,6( \pm 32,5)$ & 0,018 \\
\hline $\mathbf{H}$ & $41,6( \pm 24,6)$ & $57,7( \pm 29,8)$ & 0,0001 \\
\hline PP & $141,8( \pm 93,2)$ & $122,3( \pm 43,3)$ & 0,055 \\
\hline D & $6,9( \pm 10,1)$ & $13,3( \pm 17,6)$ & 0,001 \\
\hline K & $2,6( \pm 12,9)$ & $8,8( \pm 9,9)$ & 0,0001 \\
\hline Ch & $80,4( \pm 28,3)$ & $68,2( \pm 17,3)$ & 0,0001 \\
\hline
\end{tabular}

While comparing average trades of vitamins, there was identified, that there were high levels of vitamins $\mathrm{A}, \mathrm{E}$ and $\mathrm{Ch}$ in people, who suffer from chronic gastritis. But there was a low level of betacarotine, vitamin B5, B9, C, H, L, K in those people.

Comparing average trades of microelements in patients with chronic gastritis and control group.

\begin{tabular}{|c|c|c|c|}
\hline Microelements & $\begin{array}{c}\text { Chronic gastritis } \\
\mathbf{n = 1 2 2}\end{array}$ & $\begin{array}{c}\text { Healthy people } \\
\mathbf{n = 1 0 0}\end{array}$ & $\mathbf{P}$ \\
\hline Ca & $64,7( \pm 79,9)$ & $79,2( \pm 53,6)$ & 0,121 \\
Mg & $82,2( \pm 49,1)$ & $87,9( \pm 86,4)$ & 0,535 \\
Na & $\mathbf{1 2 0 , 3}( \pm \mathbf{3 6 , 4})$ & $\mathbf{9 7 , 4}( \pm \mathbf{3 3 , 9})$ & $\mathbf{0 , 0 0 0 1}$ \\
\hline K & $\mathbf{1 0 9 , 4}( \pm \mathbf{6 5 , 7})$ & $\mathbf{8 5 , 4}( \pm \mathbf{4 9 , 1})$ & $\mathbf{0 , 0 0 3}$ \\
\hline Ph & $\mathbf{1 4 4 , 4}( \pm \mathbf{1 0 2 , 8})$ & $\mathbf{1 1 0 , 1}( \pm \mathbf{8 7 , 7})$ & $\mathbf{0 , 0 0 9}$ \\
\hline Cl & $\mathbf{1 7 8 , 8}( \pm \mathbf{6 3 , 8})$ & $\mathbf{1 2 0 , 7}( \pm \mathbf{6 6 , 5})$ & $\mathbf{0 , 0 0 0 1}$ \\
\hline S & $71,05( \pm 34,9)$ & $65,2( \pm 32,3)$ & 0,205 \\
\hline Fe & $\mathbf{1 2 7 , 4}( \pm \mathbf{5 8 , 2})$ & $\mathbf{9 6 , 2}( \pm \mathbf{4 5 , 3})$ & $\mathbf{0 , 0 0 0 1}$ \\
\hline Zn & $77,9( \pm 37,5)$ & $67,8( \pm 33,1)$ & $\mathbf{0 , 0 3 7}$ \\
\hline
\end{tabular}




\begin{tabular}{|c|c|c|c|c|c|c|}
\hline Impact Factor: & $\begin{array}{l}\text { ISRA (India) } \\
\text { ISI (Dubai, UAE } \\
\text { GIF (Australia) } \\
\text { JIF }\end{array}$ & $\begin{array}{l}=1.344 \\
=0.829 \\
=0.564 \\
=1.500\end{array}$ & $\begin{array}{l}\text { SIS (USA) } \\
\text { PИНЩ (Russia) } \\
\text { ESJI (KZ) } \\
\text { SJIF (Morocco }\end{array}$ & $\begin{aligned}= & 0.912 \\
= & 0.234 \\
= & 1.042 \\
= & \mathbf{2 . 0 3 1}\end{aligned}$ & $\begin{array}{l}\text { ICV (Poland) } \\
\text { PIF (India) } \\
\text { IBI (India) }\end{array}$ & $\begin{array}{l}=6.630 \\
=1.940 \\
=4.260\end{array}$ \\
\hline
\end{tabular}

\begin{tabular}{|c|c|c|c|}
\hline I & $27,3( \pm 12,1)$ & $29,4( \pm 15,9)$ & 0,253 \\
\hline $\mathrm{Cu}$ & $120,3( \pm 46,6)$ & $102,4( \pm 53,2)$ & 0,008 \\
\hline Mn & $171,5( \pm 44,8)$ & $115,5( \pm 56,9)$ & 0,0001 \\
\hline $\mathbf{S e}$ & $47,5( \pm 26,7)$ & $54,1( \pm 30,01)$ & 0,087 \\
\hline $\mathrm{Cr}$ & $75,8( \pm 47,1)$ & $61,3( \pm 36,8)$ & $\mathbf{0 , 0 1 2}$ \\
\hline $\mathbf{F}$ & $11,2( \pm 31,7)$ & $21,6( \pm 24,9)$ & $\mathbf{0 , 0 0 8}$ \\
\hline Mo & $123,3( \pm 29,09)$ & $99,7( \pm 35,3)$ & $\mathbf{0 , 0 0 0 1}$ \\
\hline B & $25,1( \pm 10,6)$ & $47,2( \pm 32,1)$ & $\mathbf{0 , 0 0 0 1}$ \\
\hline $\mathbf{S i}$ & $93,07( \pm 75,7)$ & $92,3( \pm 58,4)$ & 0,933 \\
\hline Co & $177,1( \pm 52,2)$ & $114,8( \pm 43,8)$ & 0,0001 \\
\hline
\end{tabular}

Comparing average trades of microelements, there was identified, that there were statistically significantly high levels of such microelements as $\mathrm{Na}, \mathrm{Zn}, \mathrm{K}, \mathrm{Ph}, \mathrm{Cl}, \mathrm{Fe}, \mathrm{Mn}, \mathrm{Cu}, \mathrm{Cr}, \mathrm{Mo}$, Co in people, who suffer from chronic gastritis. But there was a low level of F, B microelements comparing to control group.

\section{Conclusion:}

1. As the result, there was revealed, that in $10,7 \%$ suffer from chronic gastritis. There was the most frequent prevalence of chronic gastritis in 60-74 age categories - among women

2. In people with chronic gastritis there were statistically significant trades of calories, lipids and carbohydrates, comparing to control group.
3. While comparing average trades of vitamins, there was identified, that there were high levels of vitamins $\mathrm{A}, \mathrm{E}$ and $\mathrm{Ch}$ in people, who suffer from chronic gastritis. But there was a low level of betacarotine, vitamin B5, B9, C, H, L, K in those people.

4. Comparing average trades of microelements, there was identified, that there were statistically significantly high levels of such microelements as $\mathrm{Na}, \mathrm{Zn}, \mathrm{K}, \mathrm{Ph}, \mathrm{Cl}, \mathrm{Fe}, \mathrm{Mn}, \mathrm{Cu}, \mathrm{Cr}, \mathrm{Mo}$, Co in people, who suffer from chronic gastritis. But on the other hand, there was a low level of F, B microelements comparing to control group.

\section{References:}

1. Denisova T.P., Shuldyakova V.A., Tuiltaeva L.A., Chernekov YU.V., Alipova L.N., Sadjaia L.A. (2011) Monitoring rasprostanennosti zabolevanii vnutrennyh organov na primere patologii pishevaritelnoi systemy // Saratovskii nauchno-medicinskyi jurnal. - 2011.-Tом 7, № 4.- pp. 772-776.

2. Ivashkin V.T., Sheptulin A.A., Lapina T.L., et al. (2011) Diagnostica \& lechenie funkcionalnyi dyspepsia. Gl 3 Funkcionalnaya dispepsya \& chronic gastritis/- M.: Rossiyskaya gastroenterologicheskaya associacya, 2011. - 28 p.

3. Barmagambetova AT (2007) « Clinicheskie symptomy, diagnostic, lechenie gastrita \& uroven rasprostanennosti sredi naselenie RK» UDK 616.33-002.44-006.6 (07)

4. Varbanova M, Frauenschläger K, Malfertheiner P (2014) «Chronic gastritis - an update» Best
Pract Res Clin Gastroenterol. 2014 Dec;28(6):1031-42. Copyright (C) 2014. Published by Elsevier Ltd.

5. Sipponen P, Maaroos HI (2015) «Chronic gastritis» Scand J Gastroenterol. 2015 Jun 3; 50(6): 657-667. [PubMed - indexed for MEDLINE]

6. Ströhle A, Wolters M, Hahn A. (2013) «Food supplements--potential and limits» Med Monatsschr Pharm. 2013 Sep;36(9):324-40

7. Faizulina RA, Kiasova LM, Moroz TB (2010) Nutrienty $\mathrm{v}$ sutochnom racione chronicheskom gastroduodenitom \& razlichnoi massa tela. Practicheskaya medicina №7, 2010 year. s.134

8. Ayesh MH, Jadalah K, Al Awadi E, Alawneh K, Khassawneh B (2013) Association between vitamin B12 level and anti-parietal cells and anti-intrinsic factor antibodies among adult Jordanian patients with Helicobacter pylori 


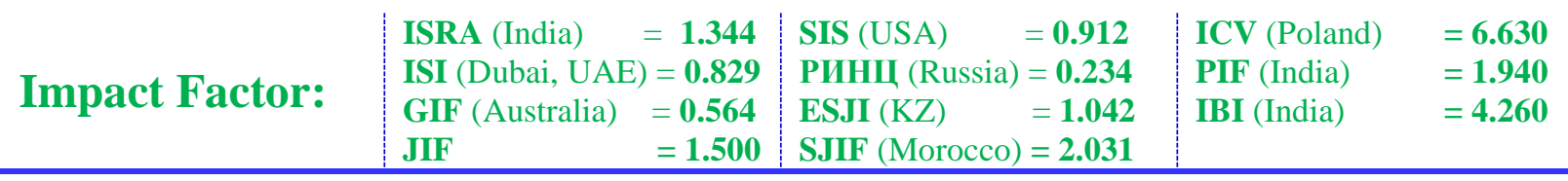

infection. Braz J Infect Dis. 2013 NovDec;17(6):629-32.

9. Naja F, Kreiger N, McKeown Eyssen G, Allard J (2010) «Bioavailability of vitamins E and C: does Helicobacter pylori infection play a role?» Ann Nutr Metab. 2010;56(4):253-9.

10. Ryz NR, Lochner A, Bhullar K, Ma C, Huang $\mathrm{T}$, Bhinder G, Bosman $\mathrm{E}, \mathrm{Wu} \quad \mathrm{X}$, Innis SM, Jacobson K, Vallance BA (2015) «Dietary vitamin D3 deficiency alters intestinal mucosal defense and increases susceptibility to Citrobacter rodentium-induced colitis». Am J Physiol Gastrointest Liver Physiol. 2015 Nov $1 ; 309(9)$
11. Assa A, Vong L, Pinnell LJ, Rautava J, Avitzur N, Johnson-Henry KC, Sherman PM (2015) «Vitamin D deficiency predisposes to adherentinvasive Escherichia coli-induced barrier dysfunction and experimental colonic injury». Inflamm Bowel Dis. 2015 Feb;21(2):297-306.

12. Kokhlova EA, Tarasova LV, Lazebnik LB (2011) «The relationship of clinical and morphological characteristics of chronic gastritis with microelements deficiency» Eksp Klin Gastroenterol. 2011;(12):36-41. 